\title{
Recycling of waste and downgrading of secondary resources in a classical type of production model
}

\author{
Eiji B Hosoda
}

Received: 15 June 2011 / Accepted: 4 April 2012 / Published online: 5 September 2012 (C) 2012 Hosoda; licensee Springer. This is an Open Access article distributed under the terms of the Creative Commons Attribution License (http://creativecommons.org/licenses/by/2.0), which permits unrestricted use, distribution, and reproduction in any medium, provided the original work is properly cited.

\begin{abstract}
This paper analyses recycling of waste and downgrading of secondary resources using a classical type of production model represented by a Sraffian model. Residuals emitted as waste by households and secondary resources obtained by recycling of waste are negatively or positively priced, depending upon technological conditions and income distribution. In other words, they can be commodities (goods) or discommodities (bads), and the distinction cannot be determined by physical characteristics. How such distinction comes about is explained, based upon an interindustrial relationship and the choice of technique. It is also demonstrated that per capita consumption may not necessarily be a decreasing function of a growth rate. This is an anomaly which cannot be found in the economy where discommodities or bads are not explicitly considered.
\end{abstract}

Keywords commodity $\cdot$ discommodity $\cdot$ recycling $\cdot$ downgrading $\cdot$ choice of technique

JEL Classification E11 $\cdot \mathrm{O} 41 \cdot \mathrm{Q} 53$

\section{Introduction}

Piero Sraffa's Production of Commodities by Means of Commodities (1960) gives us a powerful analytical tool to explore the reproducible nature of a capitalist economy. Thanks to his contribution, complicated phenomena such as capital reversal, re-switching of production technique and so on are illuminated. His method has a wide range of applicability, and environmental issues are no exception to the analytical target, although it has not been pursued at length in the Sraffian framework.

EB Hosoda $(\bowtie)$

Faculty of Economics, Keio University, 2-15-45 Mita, Minato-ku, Tokyo, 108-8345, Japan

e-mail: hosoda@econ.keio.ac.jp 
The purpose of this paper is to apply the method to the analysis of a waste management and recycling problem, which is one of the most important environmental issues. I demonstrate how the choice of technique between waste disposal and recycling affects the natural price formation of an economy, and how waste and secondary material become commodities (goods) or discommodities (bads) depending upon circumstances. I also show that the choice of technique in a market economy leads to the maximisation of per capita consumption as in the conventional model of a growing economy, if the profit rate equals the growth rate. Thus, it is shown that the golden rule of accumulation holds in this extended model. Furthermore, it is demonstrated that per capita consumption does not necessarily decrease as the growth rate increases. This is an anomaly which cannot be found in the economy where discommodities (bads) are not considered.

Although I have mentioned that the Sraffian method is not utilised sufficiently for studying environmental problems, I do not necessarily mean that there has been no contribution made by the method to the field of environmental analysis. On the contrary, quite a few researchers have contributed to this field insofar as a pure theoretical feature is concerned. Let us briefly refer to some of their works which are directly related to the present paper.

To the best of my knowledge, Franke (1986) is one of the first researchers who applied a Sraffian type of model to an environmental problem, although his model is so abstract that his work may not be regarded as being located in the stream of environmental economics. Yet, he abandons the free-disposal assumption and introduces a costly-disposal process in his paper. Apparently, this means that economic residuals, whether they be waste water, harmful gases, garbage or whatever, must be treated by a disposal process, and thus that he handles an environmental problem at an abstract level.

England (1986) also extends a Sraffian type of model on a rather earlier stage of the research history in order to present a basic model for analysing environmental problems such as air pollution, waste discharge and so on, although his treatment of the problems is very abstract, too. Using a very simple model, he shows how regulation on the emission of economic residuals affects the distribution of income, and how this type of model can be widely applicable to environmental problems.

Lager (2001) also tackles the costly-disposal problem in a more general framework. Elegantly using a linear complementarity theory, he shows that there exists a long-run equilibrium in a classical economics sense even if free disposal is restricted. A quite interesting aspect of his paper is that a distinction between commodities (goods) and discommodities (bads) is determined endogenously, so that materials become commodities (goods) or discommodities (bads) depending upon economic circumstances.

Kurz (2006) tackles a similar issue from a different angle. He surveys the traditional theory of joint production, finding that the costly-disposal problem can be handled in economics of the classical tradition. He also emphasises that there is no $a$ priori distinction between commodities (goods) and discommodities (bads).

Hosoda (2010c) analyses the same problem as Lager (2001) in a slightly different framework. Though Lager is more loyal to the original Sraffa system in adopting the assumption that the requirements for use are given, Hosoda inclines more to the analytical style of the Sraffa-Leontief-von Neumann framework. Hence, consumption 
demand is supposed to be dependent upon prices. As do other researchers, he demonstrates that the nature of a commodity (goods)/discommodity (bads) is determined endogenously in the model.

All the works mentioned above are more or less theoretical studies. Whereas they have some important conclusions for understanding the relationship between an economy and an environment, it is not so easy to deduce a concrete policy implication from those works.

Being based upon the basic Sraffian framework, Gehrke and Lager (1995) and Lager (1999) deduce quite an interesting result on environmental policy: they show that energy taxation may cause an increase in energy consumption. Although one might guess that such a perversity could happen in the economy where commodities are produced by means of commodities, they are the first to show rigorously that the perversity happens on certain conditions.

Hosoda (2010a) also applies the Sraffian framework to a more concrete environmental issue, namely the problem of waste management and recycling. He demonstrates why an upstream policy such as extended producer responsibility should be adopted to reduce household waste. He fully utilises Sraffa's idea on the distinction between basic and non-basic commodities and explains that such a policy is required only for household waste but not for industrial waste.

Since this result shows the strength of the Sraffian analysis, let me briefly explain the essence of the argument. Household-waste treatment service corresponds to a non-basic commodity in the Sraffa's sense, so that the technical condition of the service (which may be called a non-basic service), i.e. the waste treatment cost, does not affect the wage-profit frontier. Notice that the choice of technique is made depending upon the frontier in a long-run equilibrium. Thus, unless an upstream policy such as extended producer responsibility is introduced, a production process which creates more waste is adopted, since it never affects the frontier even if it is more costly.

Once the policy is introduced, waste-disposal costs are counted as production costs by producers, and the treatment service turns to be a basic-commodity (basicservice), so that the wage-profit frontier reflects the technical condition of the service. If a production process which creates more waste on the post-consumption stage is more costly, it is never chosen in an equilibrium.

The costs of industrial waste are, however, counted as part of production costs by producers, so that the waste treatment service of industrial waste is nothing but a basic-commodity (basic service), and affects the wage-profit frontier, insofar as the relevant commodity is basic. Thus, if a production process which creates more waste at the end of production is considered more costly by producers, it is never chosen in a long-run equilibrium. Hence, no upstream policy is required.

The present study follows the spirit of the above study, applying a Sraffian type of model to a very realistic feature of a recycling society where waste is recycled and recycled materials (secondary resources) are used as downgraded resources. Insofar as I know, how downgrade recycling affects price formation and per capita consumption has not been explored in a growing economy so far. I believe that this is the very issue to which the analysis based upon the classical tradition could be applied.

In the next section, the basic settings and assumptions are specified. In Section 3, the main results are presented. It is shown how waste and a secondary material become commodities (goods) or discommodities (bads) depending upon technological 
conditions and income distribution. It is also demonstrated that per capita consumption may not be a decreasing function of a growth rate when the rate is sufficiently small. The concluding remarks are given in the final section.

Although I use a classical type of production model represented by a Sraffian model, I restrict our analytical framework to a more technologically specific one in order to make model building easier and clearer: a constant coefficient production model is adopted, and duality between price and quantity systems is emphasised. One more important feature of the present study is that most of the results are demonstrated by means of examples. Hence, mathematical deductions are relegated to the appendices.

\section{The basic model and assumptions}

\subsection{A rough sketch of the model}

Let us consider an economy where two different commodities are produced by two different production techniques. The first commodity is used only as an input to production processes (i.e., a commodity used as circulating capital only), and the second one is used only for consumption. Per capita consumption $c$ is supposed to create residues or waste, which may be positively or negatively priced.

Waste can be treated by two processes, a disposal process and a recycling process. The former just disposes of waste in a harmless way, obtaining no product, whereas the latter transforms waste into a potential commodity, that is, a secondary material, which can be an alternative input to a production process of a commodity for consumption use. For simplicity, it is assumed that the secondary material cannot be used as an input to other production processes. Otherwise, things should be unnecessarily complicated.

The second commodity for consumption use (consumption goods) is, therefore, obtained by two production processes, namely the process which uses a brand-new capital commodity as an input and that which uses a secondary material as an input instead of a brand-new capital commodity. There is a difference between the two types of commodities for consumption use: the waste created by consumption of the first type can be recycled, but that of the second type cannot be, since the material embodied in the latter is supposed to be downgraded. Therefore, they are assumed to be the same as an object of consumption, but different in the post-consumption stage. It is supposed that the same amount of waste $\theta c$ is discharged from per capita consumption $c$, whether consumption comes from the first or the second type of production.

Let me give an example. Paper containers of a commodity for consumption use can be easily recycled if they are made of brand-new pulp, since the fibres contained in the paper are long and re-usable as an alternative input for brand-new pulp. On the other hand, it is not easy to recycle a used container which is made of used paper, since the fibres contained are sometimes too short to be recycled.

I do not insist that downgrading of used materials is a general character. It is easy to see that some materials such as gold, silver, platinum and other precious metals can 
be recycled without downgrading. Downgrade recycling is, however, common as seen in the example of paper recycling, and whether such recycling should be promoted or not becomes a hot issue on many occasions. This is why I analyse such recycling in this paper.

Let me remark on one more aspect of downgrading. In reality, there is gradation of downgrading of materials from perfectly recyclable quality to a non-recyclable one. Thus, downgrading is not a matter of recyclable-or-not. For instance, the recyclability of used papers is quite varied, and recycled papers can be recycled again with downgrading quality. Clearly, the most downgraded used paper must be disposed of.

Although it is desirable to reflect such gradation of downgrading quality of used materials in a model, I do not introduce such reality into the present one, since the model would be too complicated to be solved. Even a simple model which handles a recyclable-or-not nature tells us quite a few things, as shown later. ${ }^{1}$

On the nature of downgrading, another important assumption must be stated: I assume that workers or households cannot identify the different types of commodities for consumption use, i.e., commodities produced by means of a brand-new material and secondary material in the ante-consumption stage, although they may come to know the difference in the post-consumption stage. Hence, the two types of consumption commodities are priced the same.

After explaining the basic structure of an economy, I can talk about techniques in this economy. There are two techniques, i.e., $\alpha$-technique which consists of a production process of a commodity for input use, a production process of a consumption commodity which uses a brand-new input, and a disposal process; and $\beta$-technique which consists of a production process of a commodity for input use, production processes for consumption commodities which use a brand-new input and a secondary material input, and disposal and recycling processes.

The economy in which only $\alpha$-technique is adopted is easy to handle, since it is the economy where a disposal process is activated in addition to ordinary production processes. Contrary to this, an economy where $\beta$-technique is adopted is somewhat difficult to deal with. One problem is that there are two types of waste, namely recyclable and non-recyclable waste. In order to promote proper recycling, one has to sort them. With appropriate provision of information on recyclability, such as labelling, written directions, TV PR and so on, one can easily sort different types of waste. Yet, there is a possibility that such information provision is incomplete and some households may mix two types of waste, whether intentionally or not.

In this paper, perfect sorting is assumed to prevail, so that waste which is mixed up in the consumption stage is sorted perfectly in the post-consumer stage. Recyclable waste is handed over to a recycling process, and non-recycling waste to a disposal process. Although the assumption is slightly artificial, the model is still useful as a reference for understanding complicated reality. Relaxation of this assumption to accommodate imperfect sorting requires another laborious formulation. See Hosoda (2010b) in the extension of the model towards this direction.

\footnotetext{
${ }^{1}$ An aspect of gradation of downgrading could be introduced by increasing the number of equations formulated in the following. Yet, this procedure should make the mathematical treatment too complicated, and thus I do not handle the gradation problem in this paper.
} 
Table 1 The structure of production processes

\begin{tabular}{|c|c|c|c|c|c|c|c|c|c|}
\hline & $\begin{array}{l}\text { Brand-new } \\
\text { material }\end{array}$ & $\begin{array}{l}\text { Secondary } \\
\text { material }\end{array}$ & Waste & Labour & & $\begin{array}{l}\text { Brand-new } \\
\text { material }\end{array}$ & $\begin{array}{l}\text { Consumption } \\
\text { commodity }\end{array}$ & $\begin{array}{l}\text { Secondary } \\
\text { material }\end{array}$ & $\begin{array}{l}\text { Household } \\
\text { waste }\end{array}$ \\
\hline I & $a_{1}$ & 0 & 0 & $l_{1}$ & $\rightarrow$ & 1 & 0 & 0 & $\begin{array}{ll} & \\
1 & 0\end{array}$ \\
\hline II & $a_{2}$ & 0 & 0 & $l_{2}$ & $\rightarrow$ & 0 & 1 & 0 & $\theta$ \\
\hline III & $a_{3}$ & 0 & $b_{3}$ & $l_{3}$ & $\rightarrow$ & 0 & 0 & 0 & 10 \\
\hline IV & $a_{4}$ & 0 & $b_{4}$ & $l_{4}$ & $\rightarrow$ & 0 & 0 & 1 & 10 \\
\hline V & 0 & $a_{5}$ & 0 & $l_{5}$ & $\rightarrow$ & 0 & 1 & 0 & I $\theta$ \\
\hline
\end{tabular}

Here, I would like to emphasise that perfect sorting is a matter not only for households but also for recyclers. It may even be said that perfect sorting is more important for the latter, since more neatly sorted recyclable waste means better recyclability and higher profitability for recyclers. Thus, even if households do not sort the different types of waste properly, whether intentionally or unintentionally, perfect sorting is still made possible in the hands of recyclers. They may refuse to accept unrecyclable waste which is mixed up with recyclable waste. They are professional in recycling and have more information or know-how on sorting.

\subsection{Formal description of the model}

Now, let me show the model. The technology of this economy is expressed in Table 1.

The first and second rows show a process which produces a commodity for input use by means of a brand-new material, and one which produces a commodity for consumption use, respectively. The third and fourth rows show disposal and recycling processes. The fifth row shows a process which produces a commodity for consumption use by means of a secondary material.

In the second and fifth rows, there are entries of waste which are represented by $\theta$. This is because $\theta$ amount of waste is created by consumption of a unit of commodities which are produced by the second process (which uses a brand-new input) and the fifth process (which uses a secondary material as an input). ${ }^{2}$ The former waste can be treated by either the third process (the disposal process) or the fourth process (the recycling process), whereas the latter waste is treated only by the third process (the disposal process) due to the downgrading nature. Then, $\alpha$-technique is expressed by the I to III processes, whereas $\beta$-technique by the I to $\mathrm{V}$ processes. It must be noted that a disposal process is operated with a recycling process side by side in $\beta$ technique, since there are two types of waste in this economy, i.e., recyclable and non-recyclable.

In the present paper, the assumption on substitution between two types of materials is very extreme, since only simple substitution is considered: whether a secondary material is utilised as an input or not. If it is utilised, it partly substitutes for a brandnew material (an economy corresponding to $\beta$-technique). I have used the words

\footnotetext{
${ }^{2}$ I assume that an activity of waste emission as well as a consumption activity are instant for simplicity.
} 
"partly substitutes", because a secondary material cannot perfectly substitute for a brand-new material, since the latter is indispensable to obtain the former.

If one would like to take an aspect of substitution in more detail, one can do so by introducing, say, $\beta^{\prime}$-, $\beta^{\prime \prime}-, \beta^{\prime \prime \prime}$-techniques and so on to an economy whose coefficients are different from one another and reflect different combinations of brand-new and secondary materials. Even if I did so, the fundamental results should be unaffected, except that the choice of technique is on $\alpha-, \beta-, \beta^{\prime}-, \beta^{\prime \prime}-, \beta^{\prime \prime \prime}$-techniques and so on, not on an extreme choice between $\alpha$ - and $\beta$-techniques.

Let me mention another aspect of downgrading of a secondary material. As a production activity for consumption use, a process which uses a secondary material, that is, the IV process, is alternative to that which uses a brand-new one, that is, the II process. It is often seen that the former process is less productive than the latter in producing the same commodity, in the sense that more resources are used in the former than the latter. I would like to characterise the nature as $\left(a_{2}, l_{2}\right)<\left(a_{5}, l_{5}\right) .^{3}$

Let me summarise the nature of downgrading of a secondary material as follows:

\section{Assumption 1 (Downgrading property)}

1. Waste produced by consumption of a commodity which is produced by means of a secondary material cannot be recycled and must be disposed of, while that which is produced by means of a brand-new one can be recycled.

2. A production process of a consumption commodity which uses a secondary material as an input is less productive than that which uses a brand-new one, so that $\left(a_{2}, l_{2}\right)<\left(a_{5}, l_{5}\right)$ holds.

Before I give a formal description of the model, I would like to make some remarks. In the following formulation, I am loyal to Sraffa, as far as a price system is concerned: I adopt the assumption that wages are paid post factum, namely, at the end of production, and that the rate of profit is equalised by competition (Sraffa 1960). Obviously, it is possible to assume, say, that wages are advanced from capital, or that different rates of profit prevail among industries. Yet, the Sraffian tradition in these aspects should make the following analysis much easier for us.

On the other hand, as for a quantity system, I adopt the assumption which Sraffa does not adopt: I assume that all the industries grow at the same rate. I also assume that the surplus is divided into production use and consumption use. Presumably, these assumptions are harmless for an economic analysis.

Now, economies represented by the two techniques are expressed as follows.

\section{$\alpha$-technique}

Price system The price system in this economy is expressed as

$$
\left\{\begin{array}{l}
(1+r) a_{1}+w l_{1}=1 \\
(1+r) a_{2}+w l_{2}=p_{2}, \\
(1+r)\left(a_{3}+p_{3} b_{3}\right)+w l_{3}=0
\end{array}\right.
$$

\footnotetext{
${ }^{3}$ I adopt the following notation for vector inequalities. $x \equiv\left(x_{1}, \ldots, x_{n}\right) \leq y \equiv\left(y_{1}, \ldots, y_{n}\right)$ if $x_{i} \leq y_{i}$ for any $i(=1, \ldots, n) . x<y$ if $x \leq y$ and $x \neq y$.
} 
where $a_{j}, l_{j}$ and $b_{3}$ denote an input coefficient of a brand-new capital commodity and labour in the $j$ th process $(j=1,2,3)$, and a waste input coefficient in the third process (a disposal process) respectively, while $r, w, p_{2}$ and $p_{3}$ denote a profit rate, a wage rate, a price of consumption commodity and a price of waste (negative) respectively. It is assumed that $a_{1}<1$ holds. This is required for an economy to be feasible.

A brand-new material used as a circulating capital is adopted as numeraire. The first and second equations express cost-price relationships of production of commodities for circulating-capital use and consumption use respectively. The third equation is a cost-price balance of a disposal process which produces no output. Waste is inputted to the process with a negative price, and a disposal operation is completed one period after the input. Hence, the profit factor $(1+r)$ is inserted before the term $p_{3} b_{3}$ as well as $a_{3}$.

Quantity system On the other hand, the quantity system in this economy is expressed as

$$
\left\{\begin{array}{l}
(1+g)\left(a_{1} x_{1}+a_{2} x_{2}+a_{3} x_{3}\right)=x_{1}, \\
x_{2}=c \\
(1+g) b_{3} x_{3}=\theta c,
\end{array}\right.
$$

where $g$ and $x_{j}$ denote the growth rate and the activity level of the $j$ th process.

The first and second equations express the supply-demand balance of commodities for circulating capital use and consumption use respectively. The third equation is the supply-demand balance of waste. Demand for waste is supposed to come only from a disposal process.

Quantities are normalised by

$$
l_{1} x_{1}+l_{2} x_{2}+l_{3} x_{3}=1 .
$$

Thus, all the quantities, as well as consumption $c$, are normalised by the total amount of labour, and $c$ means per capita consumption.

\section{$\beta$-technique}

Price system The price system of $\beta$-technique is expressed as follows:

$$
\left\{\begin{array}{l}
(1+r) a_{1}+w l_{1}=1, \\
(1+r) a_{2}+w l_{2}=p_{2}, \\
(1+r)\left(a_{3}+p_{3} b_{3}\right)+w l_{3}=0, \\
(1+r)\left(a_{4}+p_{3}^{\prime} b_{4}\right)+w l_{4}=p_{5}, \\
(1+r) a_{5} p_{5}+w l_{5}=p_{2},
\end{array}\right.
$$

where $a_{4}$ and $b_{4}$ denote input coefficients of a brand-new material and waste for a recycling process, and $p_{3}^{\prime}$ denotes a price of waste (negative, zero or positive) respectively. A coefficient $a_{5}$ denotes the amount of a secondary material required for production of a unit of consumption commodity in the fifth process.

The first three equations are the same as those in (1). The fourth and fifth equations express a cost-price balance of a recycling process and a consumption-commodity production process which inputs a secondary material respectively. 
Quantity system Now, the quantity system in $\beta$-technique is expressed as follows:

$$
\left\{\begin{array}{l}
(1+g)\left(a_{1} x_{1}+a_{2} x_{2}+a_{3} x_{3}+a_{4} x_{4}\right)=x_{1}, \\
x_{2}+x_{5}=c, \\
(1+g) x_{4} b_{4}=\theta x_{2}, \\
(1+g) x_{3} b_{3}=\theta x_{5}, \\
(1+g) a_{5} x_{5}=x_{4} .
\end{array}\right.
$$

The first and second equations express a supply-demand balance of a brand-new material (a circulating capital-commodity) and a commodity for consumption use. The third equation expresses that waste created by consumption of a commodity which is produced by means of a brand-new material is treated by a recycling process, while that created by consumption of a commodity which is produced by means of a secondary material must be treated by a disposal process, and this is expressed by the fourth equation. The fifth equation expresses a supply-demand balance of the secondary material.

A quantity normalisation expressed by

$$
l_{1} x_{1}+l_{2} x_{2}+l_{3} x_{3}+l_{4} x_{4}+l_{5} x_{5}=1
$$

is supposed to be held.

I deal with the price system and the quantity system independently for a while. Clearly, if I add an assumption on saving behaviour, two systems are connected, so that all the systems are closed. The simplest assumption on saving behaviour is that all the profit income is saved whereas all the wage income is consumed. In this case, the famous Cambridge equation $r=g$ holds.

\subsection{Assumptions on the choice of technique}

Notice that processes I, II and III are common to both $\alpha$ - and $\beta$-techniques. The first commodity, i.e. a brand-new input being adopted as numeraire, the wage rate as well as the price of a commodity for consumption use are also common to both techniques. The price of waste is, however, not common: basically, $p_{3}$ is different from $p_{3}^{\prime}$. Apparently, the price of a secondary material $p_{5}$ appears only in the economy of $\beta$-technique.

Then, what is the principle of choice of technique in this economy? It must be noted that the wage-profit frontier is common to both techniques and is expressed as

$$
w=\frac{1-(1+r) a_{1}}{l_{1}} .
$$

Therefore, a technique cannot be chosen by utilising the frontier. This is also true even if the second commodity is adopted as numeraire.

Now, let us consider the choice of technique as follows. Suppose that $p_{3}^{\prime}>0$. Since $p_{3}$ is negative, as easily seen from the third equation of (1) or (3), I assume that $\beta$-technique is chosen, since the economy where waste can be sold is considered to be more profitable than that where waste cannot be sold and must be disposed of with charge. 
Suppose that $0 \geq p_{3}^{\prime}>p_{3}$ holds. Then, waste is negatively priced (bads) by both disposal and recycling processes. Yet, it is cheaper to treat waste by a recycling process than by a disposal process. Hence, I assume that $\beta$-technique is chosen, since the economy where waste is treated at a cheaper cost is considered to be more profitable than the other type of economy. Only when $0>p_{3}>p_{3}^{\prime}$ holds, I assume that $\alpha$-technique is chosen, since it is cheaper to treat waste in a disposal process. Let me summarise the above as follows.

Assumption 2 (Hypothesis of the choice of technique) If $p_{3}^{\prime}>0$ or $0 \geq p_{3}^{\prime}>p_{3}$ holds, $\beta$-technique is chosen. If $0>p_{3}>p_{3}^{\prime}$ holds, $\alpha$-technique is chosen.

Clearly, if $p_{3}^{\prime}=p_{3}$ holds, both techniques can be chosen, since they are equally profitable as far as waste treatment is concerned. It must, however, be noted that this criterion of the choice of technique is only on profitability of waste treatment and not on profitability of the whole economy. It will be shown later that the choice of technique based upon this hypothesis is not at all unreasonable, in the sense that the idea is a natural extension of the conventional economic model on the choice of technique in a growing economy.

I would like to explain another assumption implicitly made in (1) and (3). It is assumed that waste is priced competitively in an economy, whether it be treated by a disposal process or a recycling process. This assumption might be regarded as a little strict, since disposal of household waste is usually made by municipalities instead of private firms, so that the price of waste may not be competitively priced.

Even so, it is quite important to know the conditions of reproducibility of a capitalist economy where waste is created and must be treated. A natural price or a production price in a reproducible economy should be an important reference to a policy decision of waste management and recycling, being a guidance for municipalities.

There is another justification. Nowadays, even the service of waste treatment is being privatised gradually in many countries. Particularly, as the supply-demand balance of natural resources is getting tighter and tighter, and as the cost of municipal waste service is increasing more and more, private firms are trying to enter into this business and make profits. Therefore, it is not so far-fetched to proceed with the analysis as if the business were in the hands of private firms.

\section{The analysis and the main results}

In a linear production system which I present here, the so-called non-substitution theorem holds, so that a price system is dealt with independently of a quantity system. I, therefore, analyse the price system first.

\subsection{The analysis of a price system}

Let me show again the relationship between the profit rate and the wage rate, namely

$$
w=\frac{1-(1+r) a_{1}}{l_{1}},
$$


from which the maximum rate of profit $R$ is known as $R=1 / a_{1}-1$. This is common to both techniques. The prices of a commodity for consumption use and waste are easily calculated as

$$
\left\{\begin{array}{l}
p_{2}=\frac{l_{2}-(1+r)\left(a_{1} l_{2}-a_{2} l_{1}\right)}{l_{1}}, \\
p_{3}=-\frac{l_{3}}{(1+r) l_{1} b_{3}}+\frac{a_{1} l_{3}-a_{3} l_{1}}{l_{1} b_{3}}
\end{array}\right.
$$

from which it is easy to know that $p_{2}$ is positive and $p_{3}$ is negative. It is well known that the price of a commodity for consumption use increases (decreases) if a capitallabour ratio $\left(a_{j} / l_{j}\right)$ is larger (smaller) in the second process than in the first as the profit rate increases. This is valid here, too. On the other hand, the price of waste is shown to be increasing with respect to the profit rate. ${ }^{4}$ Thus, the waste disposal charge decreases as the rate of profit increases, since $p_{3}$ is negative.

Now, I would like to explore the price formation in $\beta$-technique. Let me deduce $p_{5}$ first before deduction of $p_{3}^{\prime}$. Clearly, $p_{5}$ is calculated as

$$
\begin{aligned}
p_{5} & =\frac{\left(l_{2}-l_{5}\right)\left\{1-(1+r) a_{1}\right\}}{(1+r) a_{5} l_{1}}+\frac{a_{2}}{a_{5}} \\
& =\frac{w\left(l_{2}-l_{5}\right)}{(1+r) a_{5}}+\frac{a_{2}}{a_{5}} .
\end{aligned}
$$

If $l_{5}$ is very close to $l_{2}, p_{5}$ is positive. Moreover, considering that $\left(a_{2}, l_{2}\right)<$ $\left(a_{5}, l_{5}\right)$, I can conclude that $p_{5} \leq 1$. Thus, the following proposition is obtained.

Proposition 1 The price of a secondary material is not greater than unity, i.e., than the price of a brand-new material for capital use, and positive if the value of $l_{5}$ is sufficiently close to that of $l_{2}$. Moreover, if both values are the same, the price is a positive constant irrespective of the profit rate and equals $a_{2} / a_{5}$. If the two values are different, the price increases as the profit rate increases.

I can show the situation in the following figure. The minimum of $p_{5}$ is calculated as

$$
\left.p_{5}\right|_{r=0}=\frac{1}{a_{5}}\left\{\left(l_{2}-l_{5}\right)\left(1-a_{1}\right)+a_{2}\right\} .
$$

This means that the price of the secondary material is positive for all the range of the profit rate if the value of $l_{5}$ is close to that of $l_{2}$, and $a_{2}$ is rather large (Figure 1a). Otherwise, the price can be negative when the profit rate is very low (Figure 1b).

Next, the price of waste in an economy of $\beta$-technique is calculated as

$$
p_{3}^{\prime}=\frac{(1+r)\left\{a_{2}-(1+r) a_{4} a_{5}\right\}+\left\{\left(l_{2}-l_{5}\right)-(1+r) a_{5} l_{4}\right\} w}{(1+r)^{2} a_{5} b_{4}} .
$$

The price of waste $p_{3}^{\prime}$ depends upon the profit rate $r$ in a complicated way. Yet, the following is true:

${ }^{4}$ This is clear from $\frac{d p_{3}}{d r}=\frac{l_{3}}{(1+r)^{2} l_{1} b_{3}}>0$. 


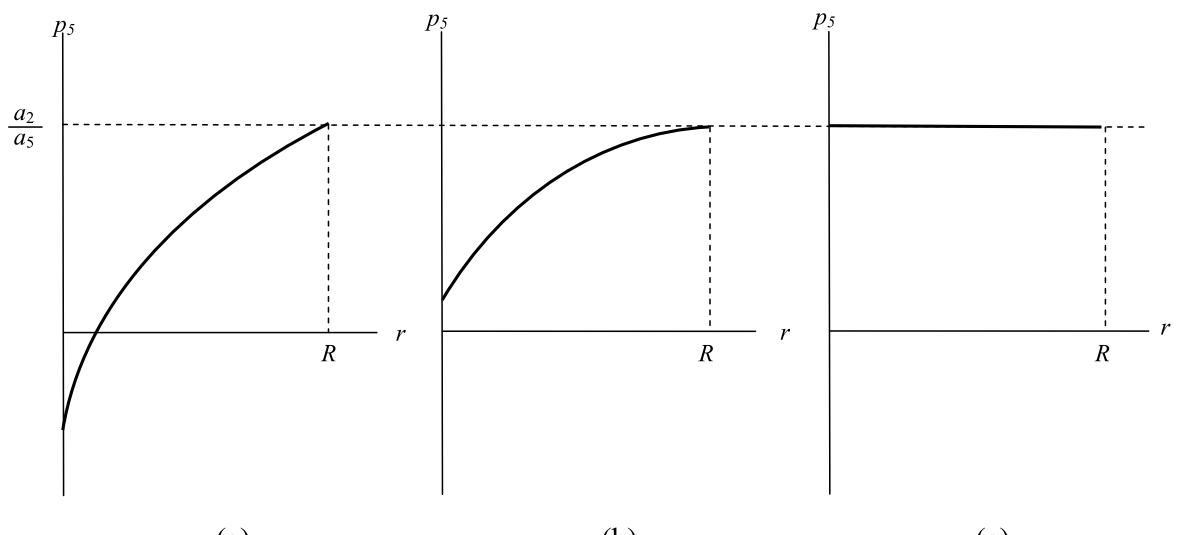

(a)

(b)

(c)

Fig. 1 (a) $l_{5}>l_{2} ; l_{5}$ is not close to $l_{2}$. (b) $l_{5}>l_{2} ; l_{5}$ is close to $l_{2}$. (c) $l_{5}=l_{2}$.

Proposition 2 Suppose $a_{2}<a_{4} a_{5}$ holds. Then, the price of waste $p_{3}^{\prime}$ is always negative.

Thus, in the case that productivity of a material input to the second process is relatively high compared to those to the fourth and the fifth processes, the price of waste is negative. The condition $a_{2}<a_{4} a_{5}$ being coupled with $l_{2}<l_{5}$, the waste input to a recycling process must be negatively priced. Negativity of $p_{3}^{\prime}$ implies that households have to pay for waste treatment service to a recycling process even if a recycled commodity or a secondary material can be sold in a market. Of course, $p_{3}^{\prime}$ can be positive, so that households may sell waste as a recyclable commodity to a recycling process.

Namely, the following holds:

Proposition 3 Suppose $a_{1} a_{2}>a_{4} a_{5}$ holds. Then, the price of waste $p_{3}^{\prime}$ is positive when the profit rate is near its maximum.

See Appendix 1 for the proof.

Thus, $p_{3}^{\prime}$ is positive if the following conditions are satisfied: (i) Material productivity of the recycling process coupled with that of the consumption-commodity production process with a secondary material input (i.e. $1 / a_{4} a_{5}$ ) is large compared to material productivity of the production process of a commodity for a brand-new material use coupled with that of the production process of a commodity for consumption use by means of a brand-new input (i.e. 1/a $a_{1} a_{2}$ ), and (ii) the profit rate is sufficiently large. Due to the assumption of downgrading $\left(a_{5} \geq a_{2}\right)$, the hypothesis in Proposition 3 implies $a_{4}$ is very small compared with $a_{1}$, meaning that the recycling process is very productive. Hence, if productivity of a recycling process is so large that the downgrading property of a secondary material is offset, $p_{3}^{\prime}$ can be positive.

What if the profit rate is very low? Is $p_{3}^{\prime}$ always negative? Not necessarily, as shown in the following proposition. 
Table 2 Signs of $p_{3}^{\prime}$ and $p_{5}$

\begin{tabular}{lll}
\hline & $(-)$ & $(+)$ \\
\hline$p_{3}^{\prime}$ & $a_{2}<a_{4} a_{5}$ & (i) $a_{1} a_{2}>a_{4} a_{5}$ and $r$ is close to $R$ \\
& $\frac{a_{2}}{p_{5}-l_{2}}<\frac{1-a_{1}}{l_{1}}$ and $r$ is close to 0 & (ii) $a_{2}>a_{4} a_{5}, a_{1}$ is close to 1 and $r$ is close to 0 \\
$p_{5}$ & $\frac{a_{2}}{l_{5}-l_{2}}>\frac{1-a_{1}}{l_{1}}$ \\
\hline
\end{tabular}

Proposition 4 Suppose $a_{1}$ is sufficiently close to unity. Then, the price of waste $p_{3}^{\prime}$ is positive if $a_{2}>a_{4} a_{5}$ holds, and the wage rate is near its maximum (the profit rate is very low).

See Appendix 1 for the proof.

From the above propositions, it is known that the price of waste treated by the recycling process in an economy of $\beta$-technique can be positive or negative, depending upon circumstances. As seen by the above propositions, it can be positive or negative, depending upon the relative magnitudes of the coefficients. Moreover, I can show that a switch of positiveness and negativeness may possibly depend upon the distribution of income.

Suppose that the hypothesis of Proposition 3 is satisfied so that $a_{1} a_{2}>a_{4} a_{5}$ holds. Furthermore, suppose that $l_{5}$ is sufficiently large. Then, the price of waste in $\beta$ technique $p_{3}^{\prime}$ is negative when $r=0$ or $w=w_{\max } \equiv\left(1-a_{1}\right) / l_{1}$, as seen from (6). Yet, it turns to positive when $r$ is near its maximum. Thus, globally, the price of waste in $\beta$-technique $p_{3}^{\prime}$ increases as the profit rate increases, although this relationship may not hold locally. Hence, both $p_{3}^{\prime}$ and $p_{5}$ increase as the profit rate increases in a global sense if the above condition is satisfied.

Table 2 shows how $p_{3}^{\prime}$ and $p_{5}$ become positive or negative. It must be noticed that $p_{3}^{\prime}$ is always negative when $p_{5}$ is negative.

Finally in this subsection, let me mention how $p_{3}^{\prime}$ changes as the profit rate increases. Although it is not easy to deduce a general rule for the movement since $p_{3}^{\prime}$ changes in a complicated way, I can show an interesting result in a special case. If a labour input to a recycling process is relatively larger than that to a production process for a brand-new material, the price of waste in $\beta$-technique $\left(p_{3}^{\prime}\right)$ increases as the profit rate increases, as shown in the following proposition.

Proposition 5 If $l_{4} \geq l_{1}$, then $p_{3}^{\prime}$ increases as the rate of profit increases.

See Appendix 1 for the proof.

\subsection{Numerical examples}

Let me show some examples which demonstrate how the price formation is made. In the following, $\theta=1$ is assumed.

Example $1 a_{1}=\frac{4}{5} l_{1}=\frac{1}{10}, a_{2}=\frac{1}{2} l_{2}=\frac{1}{2}, a_{3}=\frac{1}{2} b_{3}=\frac{1}{10} l_{3}=\frac{1}{2}, a_{4}=\frac{1}{2} b_{4}=\frac{1}{2}$ $l_{4}=1, a_{5}=\frac{1}{2} l_{5}=\frac{2}{3}$. 
When the profit rate is zero, then $w=2, p_{2}=\frac{3}{2}, p_{3}=-15, p_{3}^{\prime}=-\frac{13}{3}$, and $p_{5}=\frac{1}{3}$. Since $p_{3}^{\prime}>p_{3}$ holds, $\beta$-technique is chosen. The price of waste is negative, while that of a secondary material is positive.

Example $2 a_{1}=\frac{4}{5} l_{1}=\frac{1}{10}, a_{2}=\frac{1}{10} l_{2}=\frac{1}{2}, a_{3}=\frac{1}{2} b_{3}=\frac{1}{10} l_{3}=\frac{1}{2}, a_{4}=\frac{1}{2} b_{4}=\frac{1}{2}$ $l_{4}=1, a_{5}=\frac{1}{2} l_{5}=1$.

When the profit rate is zero, then $w=2, p_{2}=\frac{10}{11}, p_{3}=-15, p_{3}^{\prime}=-\frac{43}{5}$, and $p_{5}=-\frac{9}{5}$. Since $p_{3}^{\prime}>p_{3}$ holds, $\beta$-technique is chosen. Yet, in this case, both the prices of waste and secondary material are negative. Even so, $\beta$-technique is chosen, according to the hypothesis of the choice of technique (Assumption 1).

Example $3 a_{1}=\frac{99}{100} l_{1}=\frac{1}{10}, a_{2}=\frac{1}{2} l_{2}=\frac{1}{2}, a_{3}=\frac{1}{2} b_{3}=\frac{1}{10} l_{3}=\frac{1}{2}, a_{4}=\frac{1}{2} b_{4}=\frac{1}{2}$ $l_{4}=1, a_{5}=\frac{1}{2} l_{5}=\frac{2}{3}$.

When the profit rate is zero, then $w=\frac{1}{10}, p_{2}=\frac{11}{20}, p_{3}=-\frac{11}{2}, p_{3}^{\prime}=\frac{11}{15}$, and $p_{5}=\frac{29}{30}$. Since $p_{3}^{\prime}>0$ and $p_{5}>0$ hold, $\beta$-technique is chosen. Waste as well as a secondary material are traded as a normal commodity (goods).

Example $4 a_{1}=\frac{4}{5} l_{1}=\frac{1}{10}, a_{2}=\frac{1}{2} l_{2}=\frac{1}{2}, a_{3}=\frac{1}{2} b_{3}=1 l_{3}=\frac{1}{2}, a_{4}=1 b_{4}=\frac{1}{20}$ $l_{4}=1, a_{5}=\frac{4}{5} l_{5}=\frac{2}{3}$.

When the profit rate is $\frac{1}{4}$, then $w=0, p_{2}=\frac{5}{8}, p_{3}=-\frac{1}{2}, p_{3}^{\prime}=-10$, and $p_{5}=\frac{5}{8}$. Since $p_{3}>p_{3}^{\prime}$ holds, $\alpha$-technique is chosen, even if the price of a secondary material is positive in an economy of $\beta$-technique.

\subsection{Quantity system}

In this subsection, the quantity system is analysed. First, from (2), activity levels of $\alpha$-technique are obtained as follows:

$$
\begin{aligned}
& x_{1}=\frac{(1+g) a_{2} b_{3}+\theta a_{3}}{\left\{1-(1+g) a_{1}\right\} b_{3}} c, \\
& x_{2}=c, \\
& x_{3}=\frac{\theta}{(1+g) b_{3}} c,
\end{aligned}
$$

where $c$ denotes per capita consumption and $\sum_{i=1}^{3} l_{i} x_{i}=1$ must hold. These are the activity levels of $\alpha$-technique.

From (4), activity levels of $\beta$-technique are obtained as follows:

$$
\begin{aligned}
& x_{1}=\frac{(1+g)^{3} a_{2} a_{5} b_{3} b_{4}+\theta^{2} a_{3}+(1+g)^{2} \theta a_{4} a_{5} b_{3}}{\left\{1-(1+g) a_{1}\right\} a_{5} b_{3} b_{4}(1+g)^{2}} x_{2}, \\
& x_{2}=\frac{(1+g)^{2} a_{5} b_{4}}{(1+g)^{2} a_{5} b_{4}+\theta} c,
\end{aligned}
$$




$$
\begin{aligned}
& x_{3}=\frac{\theta^{2}}{(1+g)^{3} a_{5} b_{3} b_{4}} x_{2}, \\
& x_{4}=\frac{\theta}{(1+g) b_{4}} x_{2}, \\
& x_{5}=\frac{\theta}{(1+g)^{2} a_{5} b_{4}} x_{2},
\end{aligned}
$$

where $\sum_{i=1}^{5} l_{i} x_{i}=1$ must hold.

Coupling (7)-(9) with $\sum_{i=1}^{3} l_{i} x_{i}=1$, the activity levels of the long-run steady state of $\alpha$-technique as well as per capita consumption $c$ are obtained. In the same way, coupling (10)-(14) with $\sum_{i=1}^{5} l_{i} x_{i}=1$, those of $\beta$-technique are obtained. It is easy to see all the obtained variables are positive if $g$ is smaller than the maximum rate $(R)$. Thus, the following holds:

Proposition 6 If the growth rate is smaller than the maximum rate given by $R$, all the activity levels and per capita consumption in the long-run steady state are positive.

According to the hypothesis shown in Section 2.3 (Assumption 2), the technique is chosen on the side of the price system, namely, by comparison of $p_{3}$ and $p_{3}^{\prime}$. The quantity system follows this choice of technique. Notice I have adopted the assumption that the quality of a secondary material is downgraded compared to that of a brand-new material. Then, it might be supposed that per capita consumption in $\beta$ technique (denoted by $c_{\beta}$ ) is smaller than that in $\alpha$-technique (denoted by $c_{\alpha}$ ) due to the downgrading nature of a secondary material. Things are not so simple: the former may be larger or smaller than the latter depending upon circumstances, as shown later by means of numerical examples. Even if a secondary material is downgraded in the sense of Assumption 1, per capita consumption in $\beta$-technique can possibly be larger than that in $\alpha$-technique if productivity of a recycling process is sufficiently high.

Another interesting point on the quantity system is that there is an anomaly which cannot be seen in the conventional model, where per capita consumption is considered to decrease (increase) as the growth rate increases (decreases). This relationship does not necessarily hold in the economy where waste is disposed of or recycled. In the present model, per capita consumption may increase (decrease) as the growth rate increases (decreases) in a certain interval of the growth rate, although there is always a trade-off relationship between the wage rate and the profit rate.

To grasp the situation, let us consider the conventional multi-sectoral steady state model, where per capita consumption measured by each activity level, namely $c / x_{i}$, decreases as the growth rate increases. ${ }^{5}$ In other words, an activity level measured by per capita consumption $x_{i} / c$ increases as the growth rate increases. This implies that $\sum_{i=1}^{n} l_{i}\left(x_{i} / c\right)$ also increases as the growth rate increases. Since this term is nothing but a reciprocal number of per capita consumption, per capita consumption decreases as the growth rate increases. The important point in deducing this result is that each

${ }^{5}$ Notice that at least one $c / x_{i}$ is constant. 
activity level measured by per capita consumption increases (or does not decrease) as the growth rate increases.

This no longer holds in the present model. First, consider the $\alpha$-technique. As clearly seen from (9), the activity level of a disposal process measured by per capita consumption $\left(x_{3} / c\right)$ decreases as the rate of growth increases. The reason is as follows: if the growth rate increases, demand for a waste input to a disposal process also increases, requiring more waste than before. Thus, more per capita consumption is required insofar as the waste emission coefficient $\theta$ is constant. Consequently, if the labour coefficient of a disposal process expressed by $l_{3}$ is large compared to $l_{1}$ and $l_{2}$, per capita consumption increases as the growth rate increases. This, however, happens when the growth rate is low, and a downward relationship between the growth rate and per capita consumption is kept when the growth rate is near its maximum. This could be intuitively understood by the fact that $c=x_{2}=0\left(=x_{3}\right)$ holds when $g=g_{\max } \equiv R$ and $c>0$ when $g=0$.

The same is true for $\beta$-technique, although things are a little more complicated. Since $x_{3}$ is calculated as

$$
x_{3}=\frac{\theta^{2}}{\left\{(1+g)^{2} a_{5} b_{4}+\theta\right\}(1+g) b_{3}} c,
$$

it is easily known that $x_{3} / c$ decreases as the growth rate increases. Notice that this logic applies to an activity level of the fifth process:

$$
x_{5}=\frac{\theta}{(1+g)^{2} a_{5} b_{4}+\theta} c
$$

holds, and $x_{5} / c$ decreases as $g$ increases.

Hence, for the same reason mentioned above, per capita consumption increases as the growth rate increases if the labour coefficients of the third (a disposal) and/or fifth processes are large compared to other labour coefficients. Again, this relationship holds when the growth rate is low, and the downward relationship between the growth rate and per capita consumption holds when the growth rate is near its maximum. ${ }^{6}$

\subsection{Choice of technique and per capita consumption: numerical examples}

In the previous subsection, I have mentioned that the choice of technique is made on the side of the price system. Thus, it is natural to ask whether the choice may lead to maximum per capita consumption in a certain condition such as $r=g$. I would like to explain this by means of numerical examples. In the following examples, $\theta=1$ is supposed without loss of generality.

First, let me go back to Example 3 in Section 3.2. Namely,

$$
\begin{aligned}
& a_{1}=\frac{99}{100} l_{1}=\frac{1}{10}, a_{2}=\frac{1}{2} l_{2}=\frac{1}{2}, a_{3}=\frac{1}{2} b_{3}=\frac{1}{10} l_{3}=\frac{1}{2}, \\
& a_{4}=\frac{1}{2} b_{4}=\frac{1}{2} l_{4}=1, a_{5}=\frac{1}{2} l_{5}=\frac{2}{3} .
\end{aligned}
$$

\footnotetext{
${ }^{6}$ For a precise deduction, see Appendix 2.
} 
In this case, $\beta$-technique is chosen, since $p_{3}^{\prime}$ is positive. Easy calculation shows that $c_{\alpha}=0.0177$ and $c_{\beta}=0.0214$ when $g=0$. Per capita consumption in an economy of $\beta$-technique is larger than that of $\alpha$-technique. Choice of technique due to the hypothesis (Assumption 2) brings about larger per capita consumption.

The same is true for Example 4, i.e.

$$
\begin{aligned}
& a_{1}=\frac{4}{5} l_{1}=\frac{1}{10}, a_{2}=\frac{1}{2} l_{2}=\frac{1}{2}, a_{3}=\frac{1}{2} b_{3}=1 l_{3}=\frac{1}{2}, \\
& a_{4}=1 b_{4}=\frac{1}{20} l_{4}=1, a_{5}=\frac{4}{5} l_{5}=\frac{2}{3} .
\end{aligned}
$$

In this example, $\alpha$-technique is chosen, since $0>p_{3}>p_{3}^{\prime}$ holds. It is easy to see that $c_{\alpha}=0.6667>c_{\beta}=0.3929$. Thus, the choice of technique due to the hypothesis gives a larger per capita consumption.

Now, let us consider the following example.

Example $5 a_{1}=\frac{4}{5} l_{1}=\frac{1}{10}, a_{2}=\frac{1}{2} l_{2}=\frac{1}{2}, a_{3}=\frac{1}{2} b_{3}=\frac{1}{10} l_{3}=\frac{1}{2}, a_{4}=\frac{3}{8} b_{4}=\frac{2}{15}$ $l_{4}=\frac{1}{2}, a_{5}=\frac{4}{5} l_{5}=1$.

Suppose $r=g=0$, and $\theta=1$ again. Then, $w=2, p_{2}=\frac{3}{2}, p_{3}=-15, p_{3}^{\prime}=$ -15 and $p_{5}=-\frac{5}{8}$ are obtained. Since $p_{3}^{\prime}=p_{3}(<0)$ holds, both techniques can be chosen according to the hypothesis. Per capita consumption in both techniques is $c_{\alpha}=c_{\beta}=\frac{4}{33}$. This example suggests an interesting feature of a recycling economy. Although the waste treatment charge in the waste disposal process is the same as that in the recycling process, the price of a secondary material is negatively priced in the $\beta$-technique economy. This implies that a secondary material in this economy can be regarded as a discommodity or bads. The fifth process has a property of waste treatment in this sense. Waste discharged by households can be transformed into a commodity or goods only after the two-stage treatment. Even so, the amount of per capita consumption is the same in both economies.

The above examples allude to the fact that the choice of technique based upon the hypothesis (Assumption 2) realises the maximum per capita consumption, and that the golden rule of accumulation applies to this model. Actually, this is true in the present model. The following proposition is obtained:

Proposition 7 Suppose $r=g$. Then, $p_{3} \gtreqless p_{3}^{\prime} \Leftrightarrow c_{\alpha} \gtreqless c_{\beta}$ holds.

See Appendix 3 for the proof.

This proposition gives us justification for the hypothesis of the choice of technique stated in Assumption 2, since it is the natural extension of the conventional result on per capita consumption in a growing economy: if the profit rate equals the growth rate, per capita consumption is maximised by the choice of technique in a market.

\section{Concluding remarks}

Using a classical type of linear production model, I have analysed the economy where waste disposal and recycling activities are performed, and where the quality of a 
secondary material is downgraded, compared to a brand-new material. It is shown that prices of waste and a secondary material can be positive or negative depending upon technical conditions as well as income distribution.

Based upon the hypothesis that a technique is chosen by the comparison of prices of waste or waste treatment charges, I have demonstrated that per capita consumption is maximised in a market economy, and the golden rule holds if the profit rate equals the growth rate. This may be regarded as a natural extension of the conventional result on per capita consumption in a growing economy.

One anomaly found in the present model is that both the growth rate and per capita consumption may increase when the growth rate is low. Economic growth means an increase in the activity level of the disposal process, and this requires more waste input to the process. This effect is, however, offset by a rapid increase in the activity level of a brand-new capital-commodity production process when the growth rate is very high, since there is no room for an increase in per capita consumption, and therefore, waste.

The present model is very simple but demonstrates quite a few points which have not been explored by a mainstream economic theory. In this sense, one may say that a classical type of linear production model, represented by a Sraffian model, is powerful in investigating waste disposal and recycling problems. Further development on this line will surely contribute to daily policy-making processes of waste management.

\section{Appendix 1}

In this appendix, I show how Propositions 3, 4 and 5 hold.

Proof of Proposition 3 Suppose $r=R(w=0)$. Then,

$$
\text { Numerator of } p_{3}^{\prime}=(1+R) a_{2}-(1+R)^{2} a_{4} a_{5} \text {. }
$$

Since $1+R=\frac{1}{a_{1}}$, the above equals

$$
\frac{a_{2}}{a_{1}}-\frac{1}{a_{1}^{2}} a_{4} a_{5}=\frac{1}{a_{1}^{2}}\left(a_{1} a_{2}-a_{4} a_{5}\right) .
$$

Hence, $a_{1} a_{2}>a_{4} a_{5}$ is equivalent to $p_{3}^{\prime}>0$.

Proof of Proposition 4 Suppose $r=0\left(w=w_{\max }\right)$. Then,

$$
\text { Numerator of } p_{3}^{\prime}=\left(a_{2}-a_{4} a_{5}\right)+\left\{\left(l_{2}-l_{5}\right)-a_{5} l_{4}\right\} \frac{1-a_{1}}{l_{1}} \text {. }
$$

Hence, if $a_{1}$ is sufficiently close to unity, the second term of the above may be ignored, and $p_{3}^{\prime}$ is positive.

Proof of Proposition 5 Notice

$$
p_{3}^{\prime}=\frac{\left\{a_{2}-(1+r) a_{5} a_{4}\right\}+\left\{\left(l_{2}-l_{5}\right)-(1+r) a_{5} l_{4}\right\} \frac{w}{1+r}}{(1+r) a_{5} b_{4}}
$$


holds. Considering

$$
a_{1}+\frac{w}{1+r} l_{1}=\frac{1}{1+r}
$$

and, thus,

$$
\left(\frac{w}{1+r}\right)^{\prime}(1+r)=-\frac{1}{(1+r) l_{1}}
$$

hold, the numerator of $a_{5} b_{4}\left(d p_{3}^{\prime} / d r\right)$ is calculated as

$$
\frac{a_{5} l_{4}}{l_{1}}-a_{2}+\frac{1}{1+r}\left(\frac{1}{l_{1}}+w\right)\left(l_{5}-l_{2}\right)
$$

holds, where the denominator is $(1+r)^{2}$. Since $l_{5} \geq l_{2}$ and $a_{5} \geq a_{2}$ by hypothesis, and a strict inequality holds in either expression,

$$
l_{4} \geq l_{1} \quad \Rightarrow \quad \frac{a_{5} l_{4}}{l_{1}} \geq a_{5} \geq a_{2},
$$

which means $d p_{3}^{\prime} / d r>0$.

\section{Appendix 2}

In this appendix, I show that per capita consumption decreases as the growth rate increases when the latter is close to the maximum growth rate, whereas both may possibly increase (decrease) when the growth rate is very low. Let me start with $\alpha$ technique. Equations (7)-(9) can be rewritten as $x_{1}=\varphi_{1}(g) c$ and $x_{3}=\varphi_{3}(g) c$. Coupling these with $\sum_{i=1}^{3} l_{i} x_{i}=1$ and differentiating this, we have

$$
c^{\prime}(g)=-\frac{l_{1} \varphi_{1}^{\prime}(g)+l_{3} \varphi_{3}^{\prime}(g)}{\left\{l_{1} \varphi_{1}(g)+l_{2}+l_{3} \varphi_{3}(g)\right\}^{2}} .
$$

It is easy to show that $\varphi_{1}^{\prime}(g)>0, \varphi_{3}^{\prime}(g)<0, \lim _{g \rightarrow R} \varphi_{1}^{\prime}(g)=+\infty$ and $\varphi_{3}^{\prime}(R)>$ $-\infty$. Notice that $\varphi_{1}(g)$ contains a term $\left\{1-(1+g) a_{1}\right\}^{-1}$ and $\varphi_{1}^{\prime}(g)$ contains a term $\left\{1-(1+g) a_{1}\right\}^{-2}$ respectively. Furthermore, $\varphi_{3}(R)$ is a positive constant. Thus, we have $c^{\prime}(R)<0$, which means the downward relationship between the growth rate and per capita consumption holds when the growth rate is near its maximum. Yet, if $g$ is sufficiently small,

$$
l_{1} \varphi_{1}^{\prime}(g)+l_{3} \varphi_{3}^{\prime}(g)>0
$$

holds for sufficiently small $l_{1}$, which means that $c^{\prime}(0)>0 .{ }^{7}$ Thus, both the growth rate and per capita consumption increase when the growth rate is very small.

\footnotetext{
${ }^{7}$ It must be noted that $\varphi_{i}(i=1, \ldots, 3)$ is independent of each $l_{j}(j=1, \ldots, 3)$.
} 
Next, $\beta$-technique. (10)-(14) are rewritten as $x_{1}=\phi_{1}(g) x_{2}, x_{2}=\phi_{2}(g) c, x_{3}=$ $\phi_{3}(g) x_{2}, x_{4}=\phi_{4}(g) x_{2}$ and $x_{5}=\phi_{5} x_{2}$. Clearly,

$$
\begin{aligned}
c^{\prime}(g)= & -\left\{l_{1}\left(\phi_{1}(g) \phi_{2}(g)\right)^{\prime}+\phi_{2}^{\prime}(g)+l_{3}\left(\phi_{3}(g) \phi_{2}(g)\right)^{\prime}+l_{4}\left(\phi_{4}(g) \phi_{2}(g)\right)^{\prime}\right. \\
& \left.+l_{5}\left(\phi_{5}(g) \phi_{2}(g)\right)^{\prime}\right\} /\left\{l_{1} \phi_{1}(g) \phi_{2}(g)+l_{2} \phi_{2}(g)+l_{3} \phi_{3}(g) \phi_{2}(g)\right. \\
& \left.+l_{4} \phi_{4}(g) \phi_{2}(g)+l_{5} \phi_{5}(g) \phi_{2}(g)\right\}^{2}
\end{aligned}
$$

holds.

Set the numerator and denominator of $\phi_{1}(g)$ as $A(g)$ and $B(g)$ respectively (namely, $\phi_{1}(g) \equiv A(g) / B(g)$ ), and set the numerator of $\phi_{1}^{\prime}(g)$ as $C(g)$. Clearly, $C(g)=A^{\prime}(g) B(g)-A(g) B^{\prime}(g)$ holds. Notice $B(g)$ contains a term $\left\{1-(1+g) a_{1}\right\}$ which vanishes as $g$ goes to its maximum $\left(=g_{\max } \equiv R \equiv 1 / a_{1}-1\right)$. Then, $C(g)$ goes to

$$
\left[(1+R)^{3} a_{3} a_{5} b_{4}+\theta^{2} a_{3}+a_{5} b_{3}(1+R)\left\{(1+g) a_{4}+1\right\} \theta\right] a_{1} a_{5} b_{3} b_{4}(1+R)^{2}>0
$$

as $g \rightarrow R$. Moreover, the following is obtained:

$$
\begin{aligned}
\left(\phi_{1}(g) \phi_{2}(g)\right)^{\prime} & \\
= & \frac{(1+g) a_{5} b_{4}}{\left[\left\{1-(1+g) a_{1}\right\} a_{5} b_{3} b_{4}(1+g)^{2}\right]} \frac{1}{\left[(1+g)^{2} a_{5} b_{4}+\theta\right]} \\
& \times\left[\frac{C(g)(1+g)}{\left\{1-(1+g) a_{1}\right\} a_{15} b_{3} b_{4}(1+g)^{2}}-\frac{2(1+g) a_{5} b_{4} \theta A(g)}{(1+g)^{2} a_{5} b_{4}+\theta}\right] .
\end{aligned}
$$

Notice that $l_{1} \phi_{1}(g) \phi_{2}(g)$ in the denominator of (15) contains a term $\{1-(1+$ $\left.g) a_{1}\right\}^{-1}$ and $\left(\phi_{1}(g) \phi_{2}(g)\right)^{\prime}$ in the numerator contains a term $\left\{1-(1+g) a_{1}\right\}^{-2}$ as shown above (16), while any other $\left(\phi_{i}(g) \phi_{j}(g)\right)$ or $\left(\phi_{i}(g) \phi_{j}(g)\right)^{\prime}$ does not contain such terms, and thus those terms are bounded as $g$ goes to $R$. Therefore, only $\left(l_{1} \phi_{1}(g) \phi_{2}(g)\right)$ and $\left(\phi_{1}(g) \phi_{2}(g)\right)^{\prime}$ determine the sign of $c^{\prime}(g)$ when $g$ is very close to $R$. Since $C(R)$ is shown to be a positive constant, it is known that (15) is negative for $g$ which is sufficiently close to $R$.

Finally, it is easy to show that $\left(\phi_{3}(g) \phi_{2}(g)\right)^{\prime}<0$ and $\left(\phi_{5}(g) \phi_{5}(g)\right)^{\prime}<0$. Therefore, if $l_{3}$ and/or $l_{5}$ are sufficiently large compared to other labour coefficients, $c^{\prime}(g)$ is positive from (15). ${ }^{8}$

\section{Appendix 3}

\section{Proof of Proposition 7}

$(\Rightarrow)$ Notice that the wage rate and the price of a commodity for consumption use $\left(p_{2}\right)$ are common to both economies. Denote $x_{i j}(i=2,5$ and $j=\alpha, \beta)$ as the activities corresponding to each technique.

\footnotetext{
${ }^{8}$ It must be noted that $\phi_{i}(i=1, \ldots, 5)$ is independent of each $l_{j}(i=j, \ldots, 5)$.
} 
Taking this notation into account, let us multiply each equation of both sides of (1) by $x_{i}(i=1,2,3)$, and sum up each term in both sides of the equation. In the same way, let us multiply each equation of both sides of (2) by $p_{i}(i=1,2,3)$, where $p_{1}=1$, and sum up each term in both sides of the equation. Compare two new equations obtained by the summation, and use $g=r$. Then, we have the first equation of the following.

Similarly, multiply each equation of both sides of Eq. (3) by $x_{i}(i=1, \ldots, 5)$, and sum up each term in both sides of the equation. In the same way, let us multiply each equation of both sides of Eq. (4) by $1\left(=p_{1}\right), p_{2}, p_{3}^{\prime}, p_{3}$ and $p_{5}$, and sum up each term in both sides of equation. Compare two new equations obtained by the summation, and use $g=r$. Then, we have the second equation of the following.

$$
\left\{\begin{array}{l}
w+p_{3} \theta x_{2 \alpha}=p_{2} x_{2 \alpha}, \\
w+\left(p_{3}^{\prime} \theta x_{2 \beta}+p_{3} \theta x_{5 \beta}\right)=p_{2}\left(x_{2 \beta}+x_{5 \beta}\right) .
\end{array}\right.
$$

From these, we have

$$
\left(p_{2}-\theta p_{3}\right) x_{2 \alpha}=p_{2}\left(x_{2 \beta}+x_{5 \beta}\right)-\theta\left(p_{3}^{\prime} x_{2 \beta}+p_{3} x_{5 \beta}\right) .
$$

Since $p_{2}-\theta p_{3}>0$ holds,

$$
p_{3} \gtreqless p_{3}^{\prime} \quad \Leftrightarrow \quad c_{\alpha}=x_{2 \alpha} \gtreqless x_{2 \beta}+x_{5 \beta}=c_{\beta}
$$

is obtained.

$(\Leftarrow)$ Suppose $c_{\alpha}=x_{2 \alpha}>x_{2 \beta}+x_{5 \beta}=c_{\beta}$ holds. Furthermore, suppose $p_{3}<p_{3}^{\prime}$ holds at the same time. Since $w_{\alpha}=w_{\beta}$ holds, we have

$$
\left(p_{2}-p_{3} \theta\right) x_{2 \alpha}=p_{2}\left(x_{2 \beta}+x_{5 \beta}\right)-\left(p_{3} \theta x_{5 \beta}+p_{3}^{\prime} \theta x_{2 \beta}\right) .
$$

Since $-p_{3}^{\prime} \theta x_{2 \beta}<-p_{3} \theta x_{2 \beta}$ and $-p_{3}^{\prime} \theta x_{5 \beta}<-p_{3} \theta x_{5 \beta}$ hold by hypothesis, the following holds:

$$
\begin{aligned}
\left(p_{2}-p_{3} \theta\right) x_{2 \alpha} & <p_{2}\left(x_{2 \beta}+x_{5 \beta}\right)-\left(p_{3} \theta x_{5 \beta}+p_{3} \theta x_{2 \beta}\right) \\
& =\left(p_{2}-p_{3} \theta\right)\left(x_{2 \beta}+x_{5 \beta}\right) .
\end{aligned}
$$

Thus,

$$
x_{2 \alpha}<x_{2 \beta}+x_{5 \beta}
$$

is obtained, but this is a contradiction.

The same contradiction is deduced if we assume that both $c_{\alpha}=x_{2 \alpha}<x_{2 \beta}+x_{5 \beta}=$ $c_{\beta}$ and $p_{3}>p_{3}^{\prime}$ hold.

\section{Competing interests}

The author declares that he has no competing interests. 


\section{Additional information}

This paper was read at the seminars which were held in July 2010 at Kyoto University and in March 2011 at the University of Graz, and also in the first congress of the East Asian Association of Environmental and Resource Economics which was held in August 2010 in Sapporo, Hokkaido.

Acknowledgements The author is very grateful to all the participants for their comments and criticism. $\mathrm{He}$ is also grateful to anonymous referees of this journal for their comments.

\section{References}

England RW (1986) Production, distribution, and environmental quality: Mr. Sraffa reinterpreted as an ecologist. Kyklos 39:230-244

Franke R (1986) Some problems concerning the notion of cost-minimizing systems in the framework of joint production. Manch Sch LIV(3):298-307

Gehrke C, Lager C (1995) Environmental taxes, relative prices and choice of technique in a linear model. Metroeconomica 46(2):127-145

Hosoda E (2010a) Malfunction of a market in a transaction of waste - a reason for the necessity of an upstream policy in waste management. In: Vint J, Metcalfe S, Kurz HD, Salvadori N, Samuelson PA (eds) Economic theory and economic thought - essays in honour of Ian Steedman. Routledge, London, pp 234-253

Hosoda E (2010b) Sorting and recycling of waste in a neo-Ricardian model. Mimeo, Keio University

Hosoda E (2010c) Bads as joint products in a linear production system. In: Birolo A, Foley DK, Kurz HD, Schehold B, Steedman I (eds) Production, distribution and trade. Routledge, London, pp 33-61

Kurz DH (2006) Goods and bads: Sundry observations on joint production, waste disposal, and renewable and exhaustible resources. Progress in Industrial Ecology An International Journal 3(4):280-301

Lager C (1999) Perverse results of a greening of the tax system. In: Teixeira JR, Carneiro FG (eds) Economic dynamics and economic policy. DF-Brazil, Brasilia, pp 147-160

Lager C (2001) Joint production with 'Restricted Free Disposal'. Metroeconomica 52(1):49-78

Sraffa P (1960) Production of commodities by means of commodities. Cambridge University Press, Cambridge 